$$
\begin{gathered}
\text { DUEFR//3673-T } / 2 \\
\text { DOE/ER/13673--T2 } \\
\text { DE92 } 005268
\end{gathered}
$$

Eundamental Studies of Fluid Mechanics

\author{
and Stability in Porous Media
}

DE-FG03-87ER13673

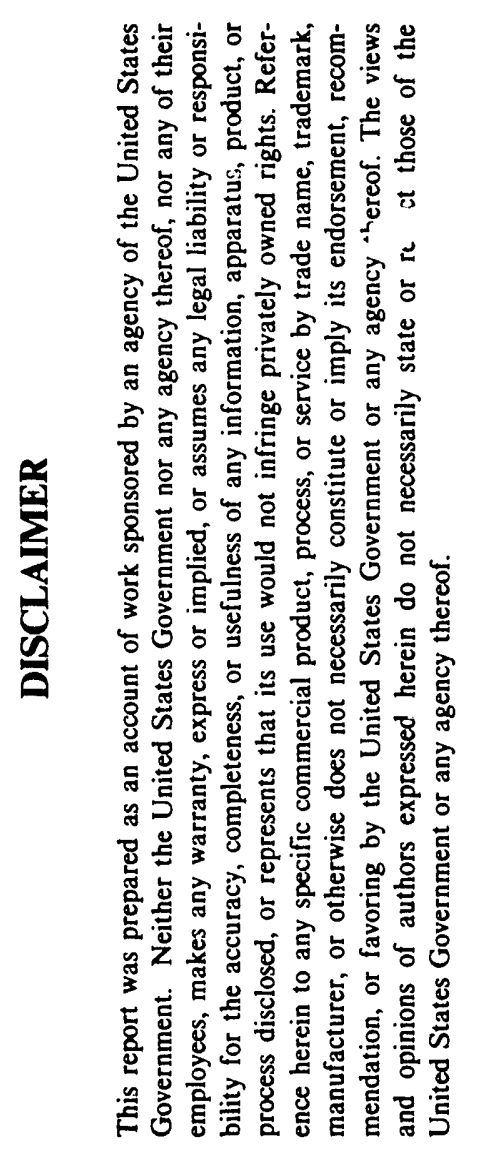

Progress Report and Continuation Request

DEC 241991

George M. Homsy

Department of Chemical Engineering

Stanford University

Stanford, CA 94305

August, 1991 


\section{Summary of Activities}

This is a summary of activities on the stated grant over the last grant period, July 1990 present. We have been active in three areas:

Numerical simulations of viscous fingering in miscible displacements

Numerical and analytical studies of the effect of non-Newtonian fluid characteristics on instabilities

Analytical studies of the onset of viscous fingering with non--monotonic mobility profiles

Progress in each of these areas is detailed in the sections below.

\section{Students}

Three PhD students have been supported or partially supported over the last grant period.

Mr. William Zimmerman - PhD candidate - Mr. Zimmerman will complete his thesis in Fall, 1991, and has been awarded a NATO Postdoctoral Fellowship for study in Madrid, Spain.

Mr. Jalel Azaeiz - PhD candidate - Mr. Azaeiz has completed all his coursework and passed the $\mathrm{PhD}$ qualifying examination. He will complete his thesis in late 1992.

Mr. Olagappan Manickam - $\mathrm{PhD}$ candidate - Mr. Manickam has completed all his coursework and passed the $\mathrm{PhD}$ qualifying examination. He will complete his thesis in late 1992.

\section{Publications}

A. Paper appearing in print during the last grant period acknowledging DOE support:

1. W. B. Zimmerman \& G. M. Homsy, Nonlinear viscous fingering in miscible displacement with anisotropic dispersion, Phys. Fluids A 3, 1859, 1991.

B. Manuscripts in preparation

1. W. B. Zimmerman \& G. M. Homsy, Large scale simulation of viscous fingering in three dimensions.

2. C.-T. Tan \& G. M. Homsy, Interaction of viscous instability with permeability heterogeneities.

3. O. Manickam \& G. M. Homsy, Fingering instabilities with non-monotonic mobility profiles. 
Invited Plenary Lecture

"Fingers, Dendrites and Cracks: Modelling Unstable Growth Processes".

SIAM Annual Meeting, July 1990, Chicago II

\section{Presentations}

G. M. Homsy "Fingers, Dendrites and Cracks: Modelling Unstable Growth Processes". Departmental Seminar, Dept. Chemical Engineering, Univ. Florida, March, 1991.

G. M. Homsy "Fingers, Dendrites and Cracks: Modelling Unstable Growth Processes". Applied Mathematics Colloquium, Stanford University, April, 1991.

O. Manickam \& G. M. Homsy "Effect cf Non-monotonic Viscosity Profiles on the Stability of Miscible Displacements in Porous Media" to be presented at the Annual Meeting of the APS Division of Fluid Dynamics, Tempe AZ, November 1991.

J. Azaiez \& G. M. Homsy "Linear Stability of Inviscid non-Newtonian Free Shear Layers" to be presented at the Annual Meeting of the APS Division of Fluid Dynamics, Tempe AZ, November 1991.

G. M. Homsy \& W. B. Zimmerman "Viscous Fingering in 3D Porous Media: A Numerical Study" to be presented at the Annual Meeting of the APS Division of Fluid Dynamics, Tempe AZ, November 1991.

\section{Work Accomplished}

\section{Viscous Fingering in Miscible Displacements (Will Zimmerman)}

Miscible displacement is of much practical interest due to its applications in enhanced oil recovery, channelling in packed beds, and fixed bed reactor regeneration. Much past work has dealt with the effects of adverse viscosity ratio and isotropic dispersion on the stability and nonlinear evolution of viscous fingers. Largely ignored have been the effects of the tensor character of dispersion and the majority of numerical work has been done in two dimensions. On the other hand, all laboratory experiments and field applications involve anisotropic dispersion and three dimensional flow. It is our intent to describe, by means of large scale numerical simulation, the effects of anisotropic, velocity dependent dispersion on the three-dimensional evolution of viscous fingers. Large scale numerical simulations also yield temporally and spatially averaged profiles which accurately predict average composition and evolution rates of the instability.

Given the complicated nature of the physical effects we are investigating, the most illuminating strategy of attack is to simulate miscible displacement with several different models, each incorporating a different component of the tensor character of dispersion. We have successfully completed a study of anisotropic Taylor dispersion effects in the 2-D evolution in the Hele-Shaw cell, Zimmerman \& Homsy (1991). We discovered several new mechanisms of finger interaction - the coalescence of multiple fingers into one larger, wider finger and the fading or pinching off of some long fingers that slow up. The most striking result was that weak transverse dispersion makes little difference in the global rate at which the instability bypasses the fixed, more viscous fluid. 
Tan \& Homsy (1988) previously considered weak enough longitudinal dispersion that an instability at the tip of fingers can develop from crossflow. Because of advances in our simulation technique, it is now possible for us to consider cases with extremely weak longitudinal dispersion. At the weakest cases of longitudinal dispersion, fingers tip-split multiple times. Shown in Figure 1 are results for very low levels of dispersion (Peclet number $=8000$ ). This is the first such numerically accurate simulation of multiple tip-splitting. Even with the complicated configuration of fingers caused by multiple tip-splitting, the average rate of finger propagation is again insensitive to $\mathrm{Pe}$.

Another problem under study is the effects of three-dimensionality on the evolution of viscous fingers. Until now there have been no reliable numerical simulations of fingering at high $\mathrm{Pe}$ in three dimensions. We have developed a new vorticity formulation that allows efficient use of spectral methods in three dimensions. Shown in Figure 2 is the evolution of an isoconcentration surface that clearly demonstrates the splitting at the tip of a finger. In the region of parameter space accessible by our study, we found little change in the average profiles from the corresponding 2-D model. Similarly, we found the 3-D interaction mechanisms of coalescence, shielding, spreading, and tip-splitting that differed little from the 2-D counterparts. We are investigating via simulation the pattern selection due to nonlinear effects among disturbances of the same wavenumber but differing in symmetry. We have shown that cellular structures with hexagonal symmetry outstrip those with square symmetry and roll symmetry by weak nonlinear self interactions, explaining the compact tubular structure of the viscous fingers shown in Figure 2.

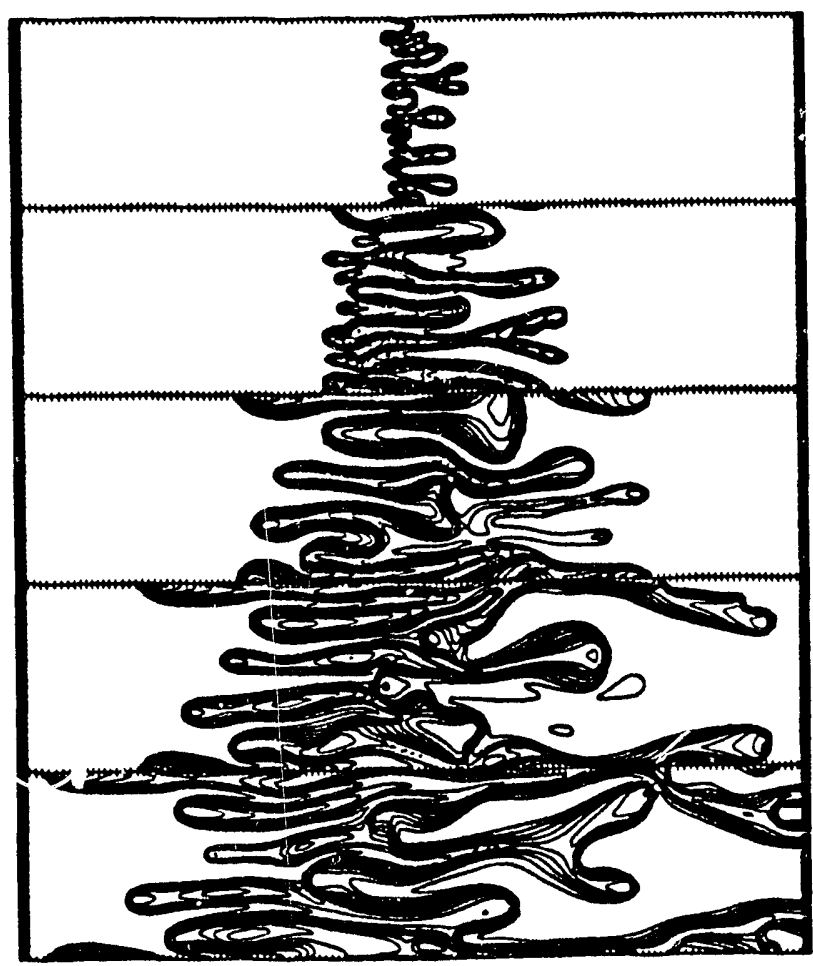

$$
\begin{aligned}
& t=584 \\
& t=1224 \\
& t=1864 \\
& t=2504 \\
& t=3144
\end{aligned}
$$

Figure 1. Simulation results for fingering at high Peclet number $(\mathrm{Pe}=8000)$ for isotropic dispersion, showing concentration contours at different times. Note the occurence of multiple tipsplitting events. Such events are often seen in experiments, and have been simulated accurately here for the first time. 


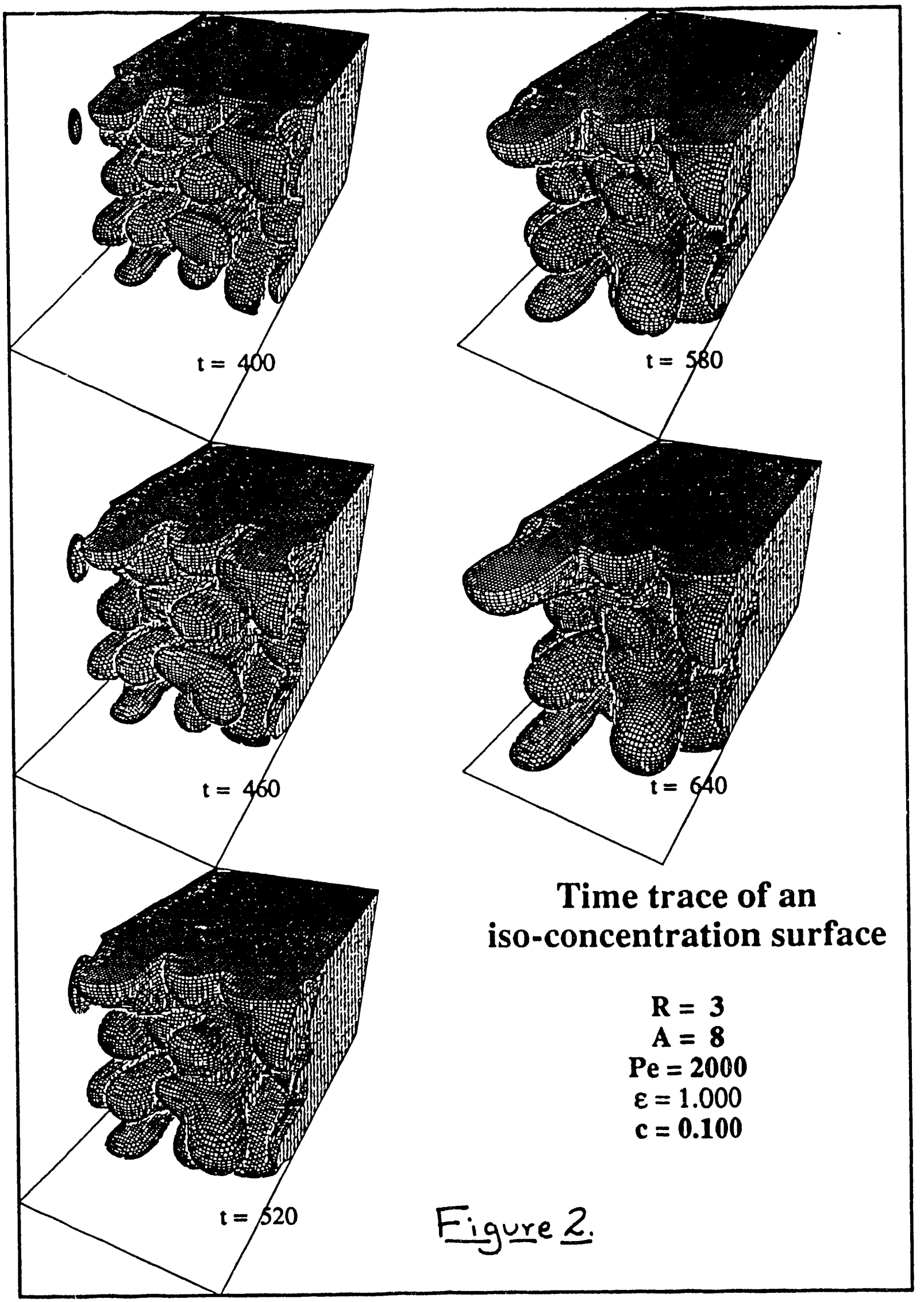




\section{Polymer Elow Interactions in Free Shear Lavers of Viscoelastic Eluids (Jalel Azaiez)}

The problem of polymer conformation and interactions with flow is key in understanding the use of polymer additives to stabilize displacements against fingering instabilities. Due to the geometrical complexity of porous media, however, the local flow field is not well-characterized, even in a statistical sense. Our interest is in understanding how the large resistance of dissolved polymers to extensional flows leads to a modification of those flows, as well as the observed increase in pressure drop through porous materials.

As a first step, we are studying these interactions in a well-defined flow, namely a free shear layer. The free shear layer represent an important prototype problem that is reasonably well-understood for Newtonian fluids (see e.g. Corcos \& Lin (1984), Metcalfe, Orszag ci al (1987), Moser \& Rogers (1991) and the many references therein). Important understanding of the mechanisms of vorticity production, subharmonic (pairing) instabilities, and vortex stretching has come from such studies.

We have recently developed a two-dimensional Hartley Spectral Navier Stokes code, and have verified it through direct simulation of the two dimensional mixing layer at Reynolds numbers ( $R e$ ) up to 400 . These validation runs were done using a small workstation. Figure 3 shows some typical results from this verification. We then modified this code to treat a specific viscoelastic model based on the Oldroyd-B constitutive equation. This model introduces a new dimensionless group referred to as the Weissenberg number (We). Both codes have been implemented on the CRAY Y-MP at the SDSC.

We have also analyzed the 2-D linear stability of a plane mixing layer in the distinguished limit of $\operatorname{Re} \Rightarrow \infty, \mathrm{We} \Rightarrow \infty, \mathrm{We} / \mathrm{Re}$ fixed. This is illustrative of the effect that viscoelasticity can have on the inviscid modes and will shed light on the mechanisms of coupling between vorticity and normal stresses associated with straining in the flow. Our preliminary results indicate that there is such a coupling which is characterized by an elasticity parameter,

$$
\mathrm{G} \equiv \mathrm{We} / \mathrm{Re}=\lambda \mathrm{v} / \delta
$$

representing the ratio of elastic to viscous relaxation times within the fluid. Analysis of the linearized equations, (not given here) indicate that the flow is governed by a modified Rayleigh stability equation. Figure 4 gives some typical results for the dispersion relation obtained by solving this modified Rayleigh equation, with $\mathbf{G}$ as a parameter. It is clear that viscoelasticity has an effect on the inviscid modes, which we believe is due to the plane strain associated with the Kelvin-Helmholtz instability. 

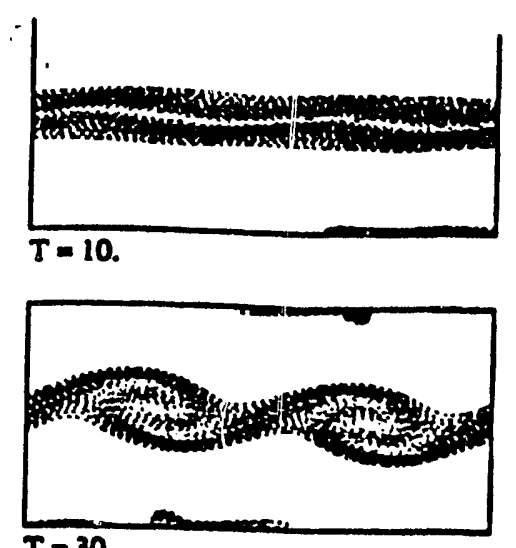

$T=30$.

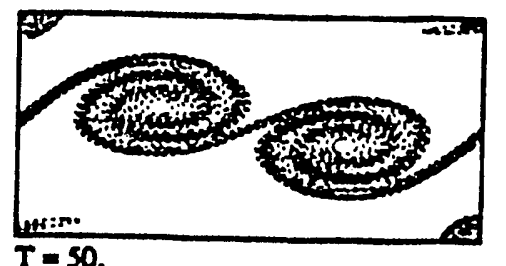

$T=50$.

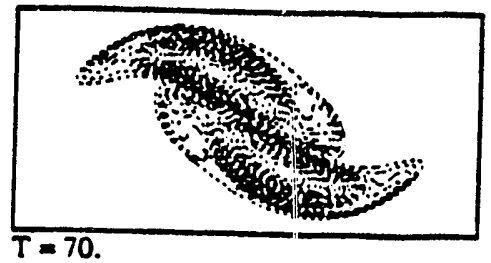

$R \theta=400$. ALPHA $=0.445$
Eiqure 3.

Roll-up and pairing for a Newtonian fluid with $R e=400$. Comparison with previous results validates our Hartley spectral code.

\section{VORTICITY CONTOURS (PAIRING)}

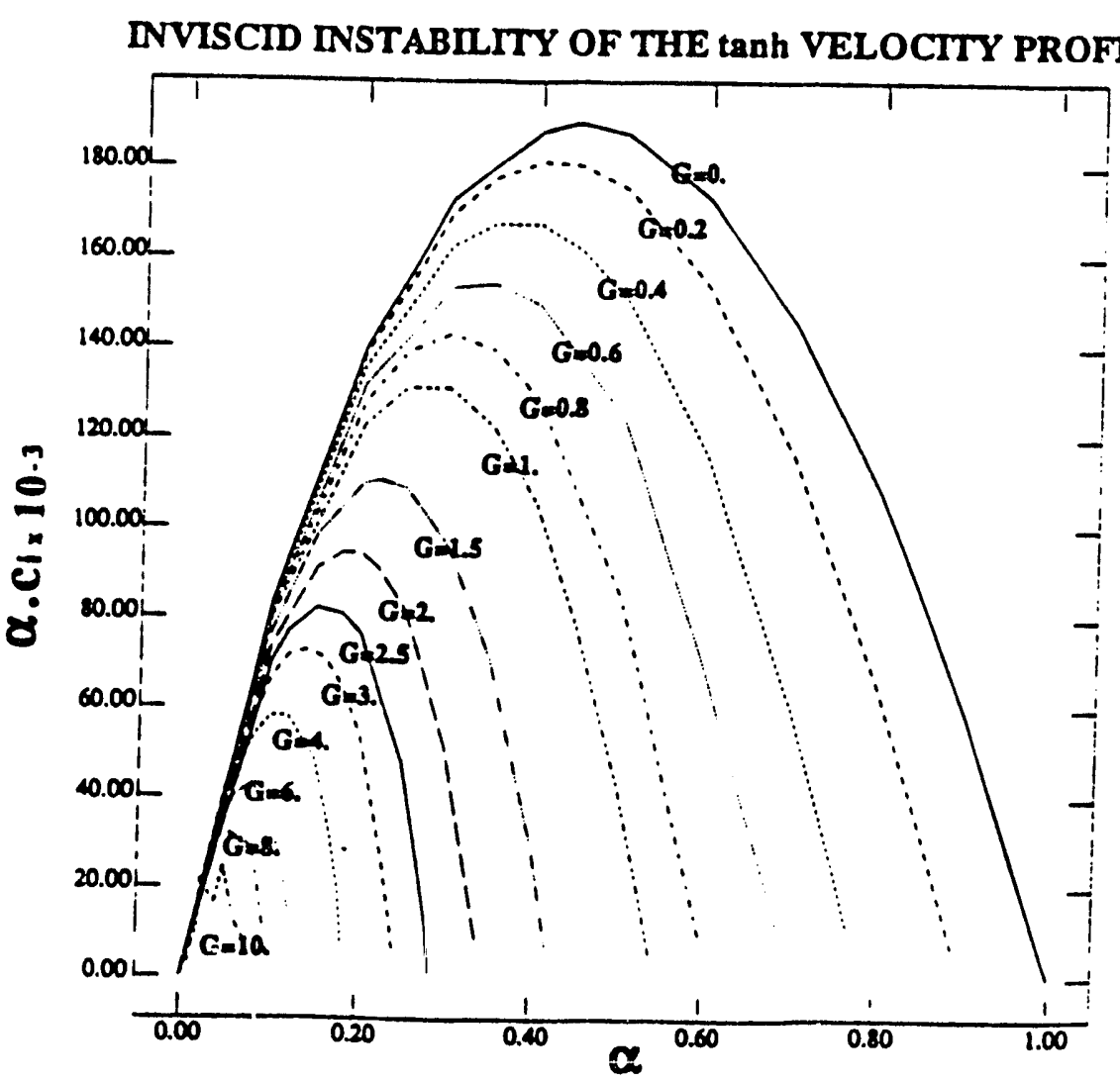

\section{Eigure.4.}

Temporal Growth rate vs. wave number with $G$ as a parameter: from solution of modified Rayleigh equation obtained by taking $\operatorname{Re} \rightarrow \infty$, We $\rightarrow \infty$. We/Re fixed. $G=0$. is the classical inviscid limit. 
Effect of Non-monotonic Viscosity Profiles on the Stability of Miscible Displacements in Porous Media (O. Manickam)

We consider the miscible displacement process illustrated below.

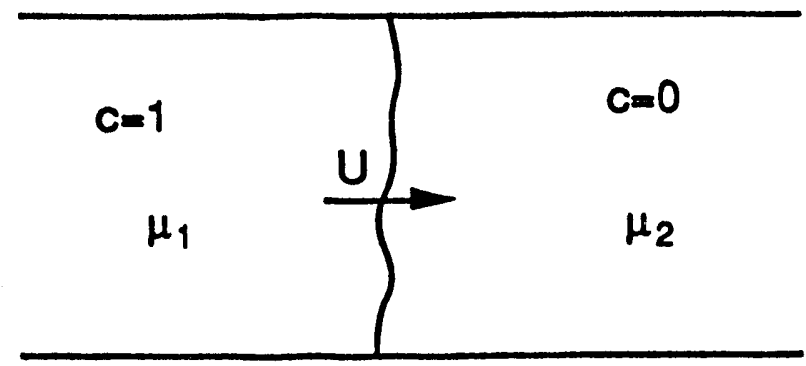

A fluid of viscosity $\mu_{1}$ displaces a fluid of viscosity $\mu_{2}$ at a uniform base velocity $U$. Such miscible displacement processes are of interest in enhanced oil recovery where for example $\mathrm{CO}_{2}$ is used to displace oil out of the reservoir. The viscosity $\mu$ of the fluid depends on the local composition c. For a monotonic dependence of viscosity on concentration $\mu(c)$ it is known that an unfavourable viscosity contrast $\left(\mu_{1}<\mu_{2}\right)$ results in instability. The objective of the present work is to study the effect of non-monotonic viscosity profiles on the stability of the interface.

As a first step we have employed a linear stability analysis to study the stability of the process. We have obtained an analytical solution when the base state concentration is a step profile. Further analytical solutions are possible in the form of asymptotic expansions of the growth rate for short times and for small wave numbers.

The results indicate that the stability of the interface depends not on the end-point viscosities but on the end-point derivatives of viscosity with respect to concentration. The criteria for instability is $(\mathrm{d} \mu / \mathrm{dc})_{\infty 1}+(\mathrm{d} \mu / \mathrm{dc})_{\infty}<0$. This gives the following interesting possibility. A non-monotonic variation of viscosity with concentration $\mu(c)$ can yield a stable displacement even if the viscosity contrast is unfavourable $\left(\mu_{1}<\mu_{2}\right)$. Asymptotic expansions for short time shows that the diffusion of the base state does not always mitigate the instability. If $\mu(c)$ is non-monotonic the diffusion of the base state can in fact enhance the instability.

\section{Proposed Work}

During the next grant period we intend to pursue the projects discussed above. Mr. Zimmerman's work is quite mature and will be finished within the next 3 months. The work of Mr. Azaiez and Mr. Manickam are in roughly equivalent stages of development, and their course over the next year is fairly well-set.

\section{Yiscous Eingering in Miscible Displacements (Will Zimmerman)}

The remaining work on this project is to focus in more detail on the effects of the velocity dependence of dispersion on the propagation of the instability. We intend to simulate miscible displacement with a more general velocity dependence than Taylor dispersion. In addition, we will finish the analysis of the three dimensional simulations, prepare a manuscript for publication, and compare both our two- and three-dimensional results with the experimental results of Professor Dominique Salin (Paris VI). 
We propose to pursue the linear stability analysis of the plane mixing layer in order to study the detailed interaction between the evolution of vorticity and the extra stresses carried by the polymer. We will analyse numerically the secondary instability of the equilibrated vortical structures using standard techniques of linear stability theory. These studies will be complemented by large scale numerical simulations in two and three dimensions.

Effect of Non-monotonic Viscosity Profiles on the Stability of Miscible Displacements in Porous Media (O. Manickam)

Linear stability analysis gives the possibility of a stable displacement even under an unfavorable end-point viscosity contrast. But this holds true only for small disturbances. It is not clear whether the displacement will remain stable when the disturbances are of finite amplitude. Further we suspect that the non-monotonicity of $\mu($ c) will give rise to "penetrative fingering", in which the spatial extent of the fingering will be limited to 'unfavorable' parts of the mobility profile, and therefore will not propagate indefinitely as occurs with monontonic profiles: see e.g. Figure 1. In order to investigate these issues we plan to apply the bifurcation theory to our problem.

The next step is to study the effect of non-monotonic viscosity profiles on the observed averaged features of viscous fingering. To study this we need to numerically simulate the miscible displacement process. The simulation will use a Hartley transform based numerical scheme and will closely follow that of Zimmerman \& Homsy (1991).

\section{References}

Corcos, G. M. \& S. J. Lin "The mixing layer: deterministic models of a turbulent flow: Part 2. The origin of the three-dimensional motion" J. Fluid Mech. 130, 67, 1984.

Metcalfe, R.W., S.A. Orszag, M.E. Brachet, S. Menon \& J.J. Riley "Secondary instability of a temporally growing mixing layer" J. Fluid Mech. 184, 207, 1987.

Moser, R. D. \& M. M. Rogers "Mixing transition and the cascade to small scales in a plane mixing layer" Phys. Fluids A, 3, 1128, 1991.

Tan, C.-T. \& G. M. Homsy "Simulation of nonlinear viscous fingering in miscible displacement" Phys. Fluids 31, 1330, 1988.

Zimmerman, W. \& G. M. Homsy "Nonlinear viscous fingering in miscible displacement with anisotropic dispersion" Phys. Fluids A 3, 1859 (1991). 

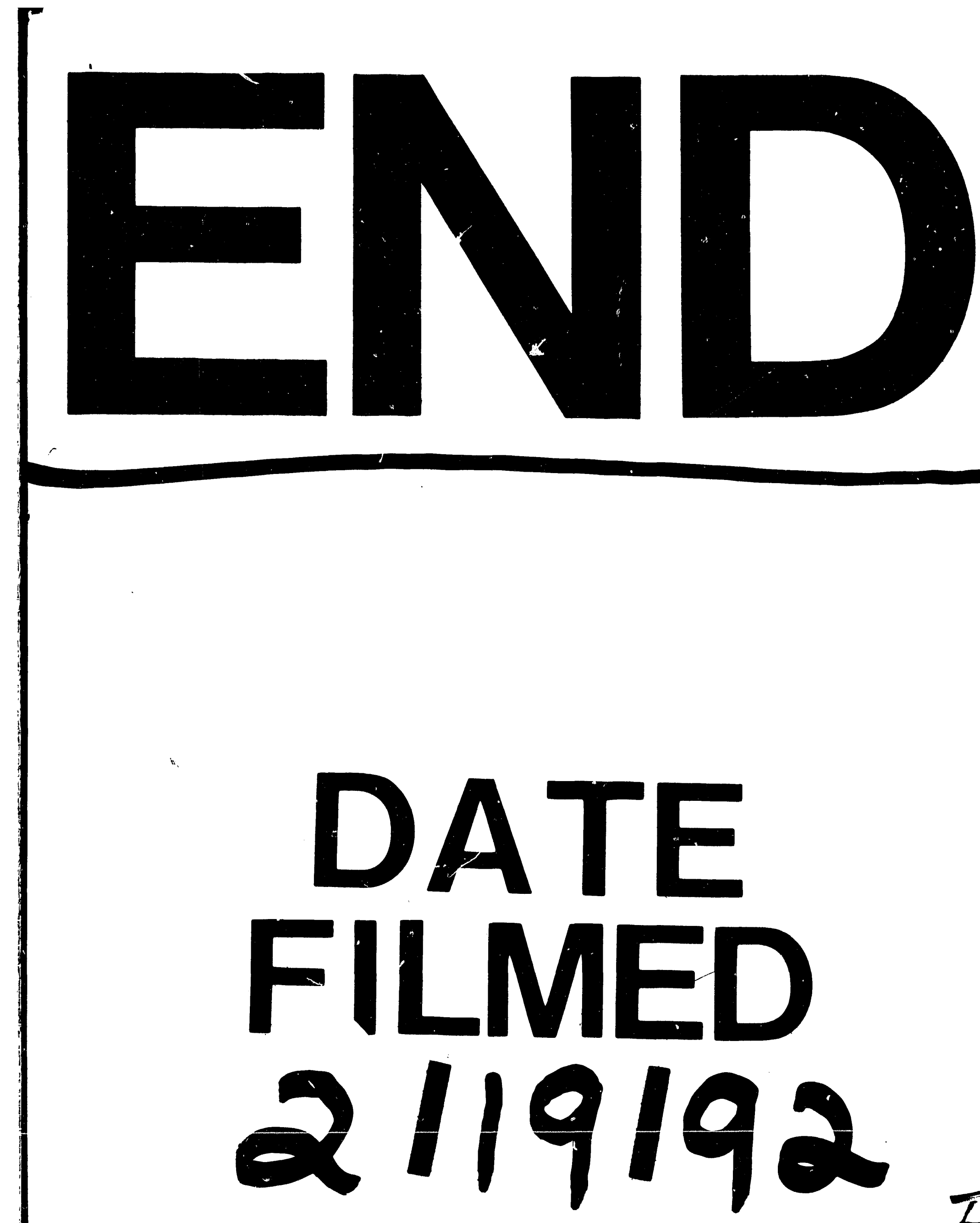

$I$ 


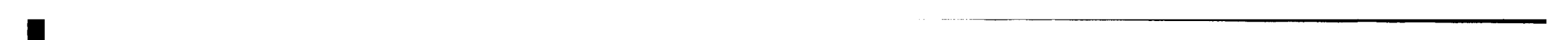

